\title{
Tensioned Metastable Fluid Detectors in Nuclear Security for Passively Monitoring of Special Nuclear Materials-Part A
}

\author{
Tom Grimes", Rusi Taleyarkhan \\ Purdue University, West Lafayette, USA \\ E-mail: "tfgrimes@purdue.edu, rusi@purdue.edu \\ Received June 22, 2011; revised July 23, 2011; accepted August 27, 2011
}

\begin{abstract}
This paper (constituting Part A) describes the transformational Tensioned Metastable Fluid Detector (TMFD) based method for "passive" detection of Special Nuclear Materials (SNMs) as related to nuclear security. Purdue University is developing novel, multi-purpose tension metastable fluid nuclear particle detectors by which multiple types of nuclear particles can be detected with high $(90 \%+)$ intrinsic efficiency, spectroscopic capability, directional information, rapid response, large standoff and significant cost-savings compared with state-of-the-art systems. This paper focuses specifically on recent advances in the use of these novel detector systems for neutron spectroscopy. These techniques will then be discussed and evaluated in the context of area monitoring in waste processing applications with a focus on passive monitoring of radioactive source particles from SNMs. The companion paper (Part B) addresses TMFD technology as it pertains to active interrogation.
\end{abstract}

Keywords: Neutron Detection, Metastable, CTMFD, ATMFD, Spectrometry, Unfolding

\section{Introduction}

The current global security and nuclear proliferation climate has introduced a need for game-changing detectors to fill specific needs in the global security landscape [1]. Two of the most pressing needs are for real time monitoring of ports and monitoring of spent fuel reprocessing facilities.

For the vast majority of situations that require real time monitoring, unless the SNM in question is cleverly masked so that tell-tale nuclear emission signatures, especially neutrons from spontaneous fission are not readily available for scanning, passive detector systems are relied upon. In a variety of situations pertaining to nuclear security (e.g., scanning baggage at various points of transfer, safeguards-related assays and material balances, as well as for monitoring for Pu-based isotopes in spent nuclear fuel reprocessing systems) it is especially, and extremely desirable to have access to passive neutron detectors with spectroscopic and directionality capabilities that are of high intrinsic efficiency, with gamma photon insensitivity (a feature of interest also for active interrogation), and which provide acceptably low false- positives, e.g., from the well-known "ship effect" arising from cosmic particle-related interference radiation, in order to successfully detect and interdict SNM material being smuggled in shipping containers.

Monitoring of spent fuel reprocessing streams [2,3] is another application that requires transformational advances in detector technology. In the current technological climate there is a clear and present fear that diversion (particularly of $\mathrm{Pu}$ isotopes) could be done (in the $8+\mathrm{kg}$ range from large processed inventories in the $1,000+\mathrm{kg} / \mathrm{y}$ range) without detection. Stopping such an attack requires advanced real-time monitoring passive detection techniques for material accountability. The envisaged safeguard techniques employ metastable fluid detectors equipped with neutron spectrometry as area monitors as well as complementary systems composed of metastable fluid detectors fed material by sipping techniques and employing alpha spectrometry to monitor the waste streams directly. In such an environment gamma insensitivity is of paramount importance as the fluence of gammas is enough to blind commonly used detectors (e.g., $\mathrm{He}-3, \mathrm{LiI}, \mathrm{BF}_{3}$ or NE-213) that are even partially sensitive to them [4]. Material accountability in this environ- 
ment specifically requires spectrometry because noticing changes in overall radioactivity is more difficult than noticing changes in the select characteristic emissions from the specific isotopes of interest.

Briefly [5,6] a liquid in a tension metastability fluid detector (TMFD) becomes sensitive to radiation, for example neutrons, when the metastable liquid is in a subzero (i.e., below vacuum pressure) fluid state. Particle interactions in TMFDs result in audible, visible bubble bursts that can not only be heard and seen by the naked eye, but also be recorded and analyzed using conventional electronic counting-analyzer systems. It will be shown that the degree of metastability can be correlated to radiation type, (e.g., alphas, fission products, neutrons, photons) energy, and to the desired response time when other variables are kept constant.

In development are both the centrifugal and acoustic systems. The centrifugal system, hereafter CTMFD, brings the fluid to a tension metastable state by using the centrifugal force principle using the apparatus configuration shown in Figure 1; whereas, the acoustic system, hereafter ATMFD, puts the fluid in a metastable state with the rarefactions of an oscillating pressure field which is induced with coupled piezoelectric drivers Figure 2 [7].

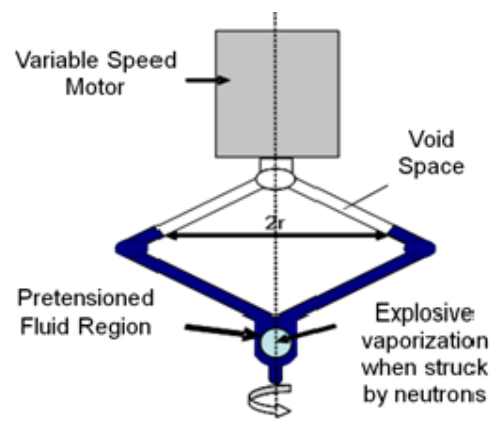

Figure 1. Centrifugally Tensioned Metastable Fluid Detector (CTMFD) [14].

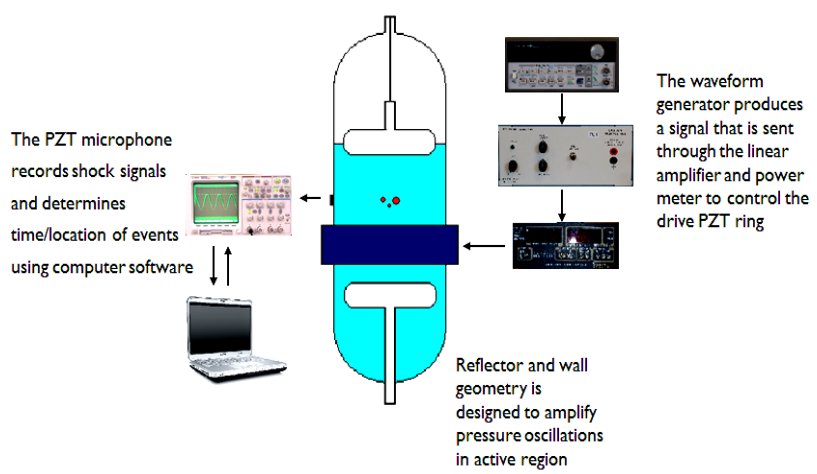

Figure 2. Acoustically Tensioned Metastable Fluid Detector (ATMFD) [16].

\section{Conventional Neutron Spectroscopy Techniques}

The approach for TMFD spectroscopy was adapted from the well-known [8] techniques that were developed for spectroscopy for use with conventional thermal neutron detectors. One of the most prevalent techniques uses "Bonner spheres". Bonner spheres are a series of polyethylene spheres that cover a thermal neutron detector [9]. The polyethylene in the spheres is designed to scatter and moderate neutrons from the $\mathrm{MeV}$ to the $\mathrm{eV}$ range. As the radius of the sphere becomes larger, neutrons from high energies are more likely to scatter down to lower energies where the detector has a very high efficiency for them. On the other hand, neutrons from very low energies will be more likely to scatter away without reaching the detector. Thermal neutron detectors such as $\mathrm{LiI}$ or $\mathrm{BF}_{3}$ detectors are exposed to the unknown neutron source repeatedly while inside a Bonner sphere of each radius (occasionally also done using $\mathrm{Cd}$ to get good resolution of the thermal neutron region or $\mathrm{Pb}$ to get better resolution in the $\mathrm{MeV}$ neutron energies). Using the information the detector records for each run, the neutron count information is combined with the "Response Matrix" of the system which contains information about the relationship between flux intensity and detector response. Using a technique called unfolding by which inverse problems are solved, a variety of algorithms can be used to determine the spectrum of the unknown neutron source. Put simply:

$$
[\mathrm{DR}]_{\mathrm{Nx} 1}=[\mathrm{RM}]_{\mathrm{NxM}} \times[\mathrm{NS}]_{\mathrm{Mx} 1}
$$

[DR] $=$ Detector response $(\mathrm{Nx} 1$ vector of measured counts at each of $\mathrm{N}$ different moderator thicknesses),

$[\mathbf{R M}]=$ Response matrix $(\mathrm{NxM})$,

[NS] = Incoming neutron spectrum (Mx1 vector of counts in each of $\mathrm{M}$ energy bins).

\section{Establishing the Response Matrix via MCNP}

Often the response matrix of a Bonner sphere system is determined experimentally by subjecting the system to a series of monoenergetic neutron sources. The well-established Monte-Carlo based nuclear particle transport code MCNP [10] allows such an assessment to be done without the need for expensive experimentation in order to find the response matrix.

In order to perform the MCNP-based assessment to represent the assessment via actual experimentation, a MCNP model must be created for each detector-moderation geometry with a series of (simulated) mono-energetic neutron sources. Once completed, the detector re- 
sponse (for instance, a multiplier tally that searches for and catalogs ( $\mathrm{n}$, alpha) reactions for a LiI detector) is gathered from each model in order to construct the response matrix.

Figure 3 shows a sample graphical representation of a response matrix for a LiI detector surrounded with various thicknesses of high-density polyethylene spheres. Each curve represents the relative number of counts taken by a detector covered by a sphere with the given radius of polyethylene across all neutron energies in semi-log scale. Response peaks at the low neutron energies for the spheres with smaller diameter because fewer of these low energy neutrons are scattered away by the relatively smaller amount of moderating material. Response peaks are noted at high neutron energies for spheres with larger diameters because the relatively larger amount of material is more efficient at reducing the energy of the incident neutrons causing an increase in detection probability due to the logarithmically increasing neutron absorption cross-section with reduced neutron energy [8]. To remain consistent with the past usage, dimensions of moderators are presented in inches.

MCNP modeling has been shown to find Bonner sphere response matrices to within acceptable tolerance by Vega-Carrillo H. R. et al. [11]; however, seldom have studies been done to assess the possibility of performing such spectroscopy with non-spherical moderation geometries. For thermal neutron detectors the creation and validation of non-spherical geometries is practically significant and interesting because it could allow one to create custom moderation geometries that could be specifically designed to fit into the space available for the detector while allowing up to a factor of 10-100 times less expense compared to costs for buying calibrated, spherically cast Bonner spheres.

One seemingly obvious approach for doing spectrometry with TMFD detectors is to use the same type of

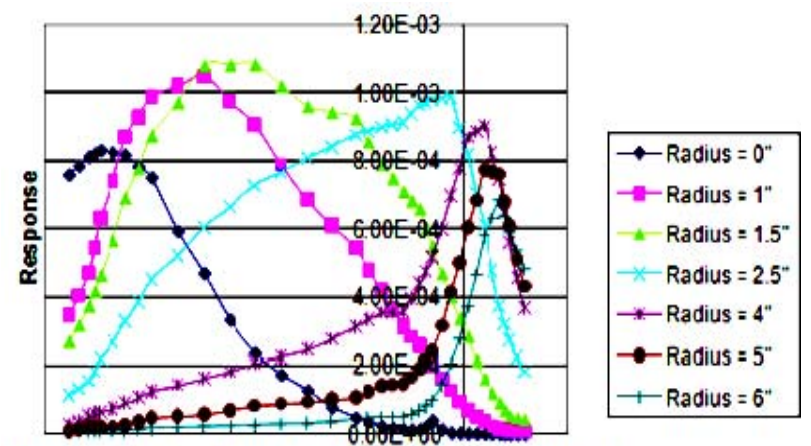

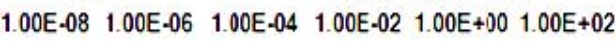

Energy (MeV)

Figure 3. MCNP5 generated response matrix for a LiI detector in "spherical" geometry (Figure 5). polyethylene moderation and do spectrometry in much the same way as it is done with thermal neutron detectors. However, contrary to conventional $\mathrm{LiI}$ and $\mathrm{BF}_{3}$ thermal neutron detector designs, the CTMFD and ATMFD systems are not amenable to being engulfed in spherical polyethylene balls. Therefore the application to TMFD systems makes the pursuit of non-conventional moderating geometries much more interesting and relevant. The development of a design framework-cum-protocol with use of non-spherical moderators was first attempted using conventional thermal neutron detectors. The first nonspherical moderating geometry attempted was the "rectangular" geometry in which 4 " by 8 " blocks of varying thickness polyethylene were placed between the detector and the source as shown in Figure 4. The same method of MCNP-based analysis was carried out for the "rectangular" geometry and a response matrix was obtained for a LiI detector. The response curves for "rectangular" geometry (Figure 6) are rather similar to those of the "spherical" geometry (Figure 3) for small and moderate amounts of shielding. However, for the higher amounts
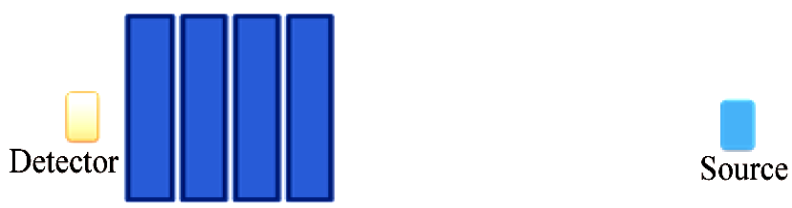

Polyethylene

Figure 4. Schematic of "rectangular" geometry.
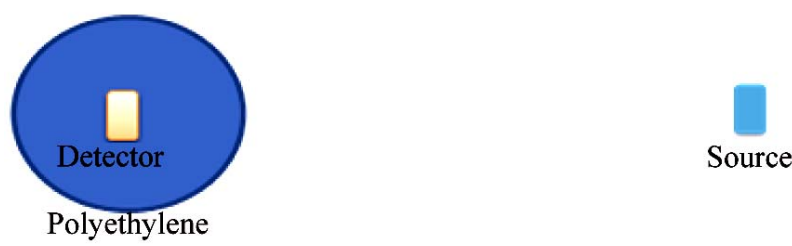

Figure 5. Schematic of "spherical" geometry.

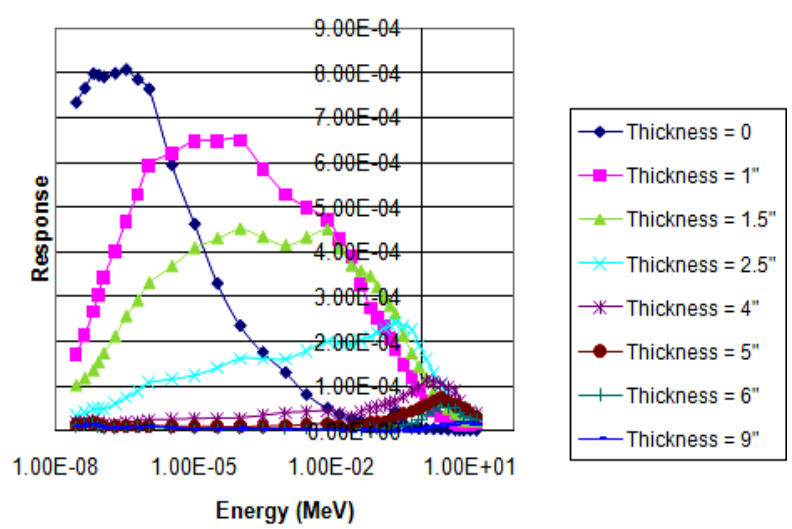

Figure 6. MCNP5 generated response matrix for a LiI detector in "rectangular" geometry (Figure 3). 
of shielding where many more down-scattering events must take place before detection the "rectangular" moderator configuration geometry is less efficient at scattering neutrons that go past the detector back to where they can be detected-an issue which was remedied with design of a "Tent" geometry.

Figure 7 depicts the "Tent" moderator geometry created to surround the detector. This geometry consists of the same sized pieces of polyethylene used to form the "rectangular" geometry shown in Figure 4, but the pieces are arranged with four walls and a top enclosing the detector in order to reflect neutrons towards the detector from multiple sides with the aim to correct the shortcomings of the rectangular geometry.

\section{Model Validation}

Validating the various MCNP-based models was conducted in step-wise fashion. As a first step, our MCNPbased calculation results for a LiI detector surrounded with Bonner spheres of various thicknesses were compared and calibrated against the already-published results of others [11]. Results of the comparison are shown in Figure 8 which shows excellent agreement except only in thermal energy bins which suffer somewhat in accuracy due to issues related to the 3-D geometry effects.
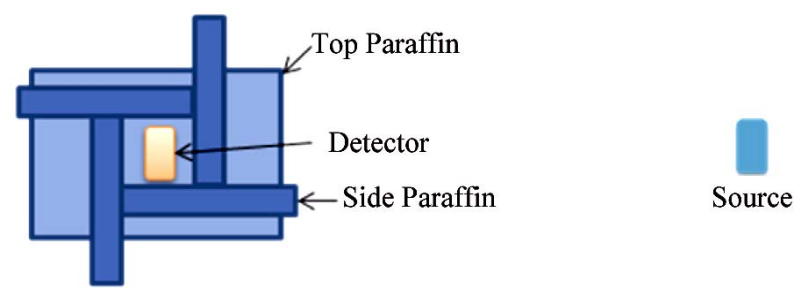

Figure 7. Schematic of "tent" geometry.

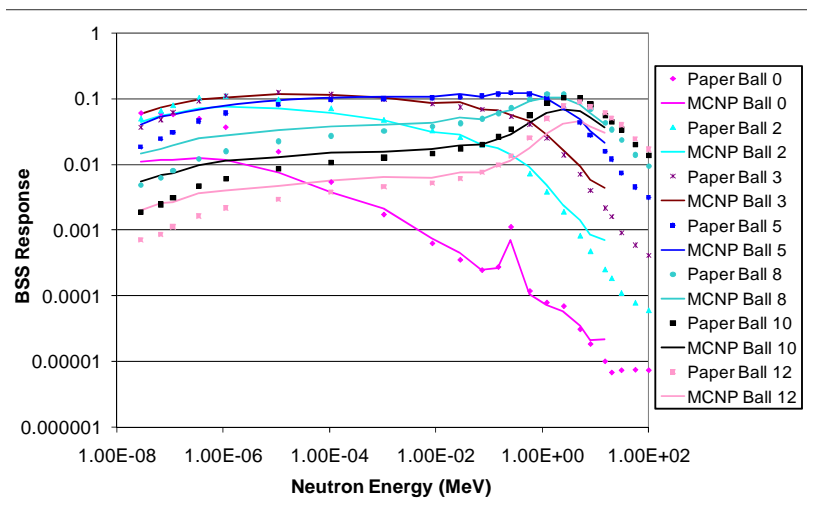

Figure 8. Response matrix of LiI detector in Bonner Spheres as calculated with MCNP5 and compared to VegaCarrillo H. R. et al. [11].
For the non-standard geometries, the validation process was much more difficult since comparable data for non-spherical moderator geometries are unavailable. The "tent" and "rectangular" configurations of Figure 4 and 7 are unique and could not be validated against results of others. Thus, it was necessary to conduct experiments with $\mathrm{LiI}$ and $\mathrm{BF}_{3}$ detectors in these configurations to validate the models. For this experimentation, the laboratory's ${ }^{252} \mathrm{Cf}$ spontaneous fission, and $\mathrm{PuBe}(\mathrm{alpha}, \mathrm{n})$ neutron sources were used.

The first and simplest step taken was to make sure the spectra obtained for different sources were distinguishable when moderated with the new moderation geometry. Using both a ${ }^{252} \mathrm{Cf}$ source as well as a Pu-Be source, tests were performed with a LiI detector and the "rectangular" geometry of Figure 4. Results are shown in Figure 9. The $252 \mathrm{Cf}$ source (as to be expected) results in higher counts for smaller moderator thicknesses because the neutrons that are emitted by this source have a most probable energy of $\sim 0.8$ $\mathrm{MeV}$ versus $\sim 4 \mathrm{MeV}$ from the $\mathrm{Pu}-\mathrm{Be}$ source.

Figure 10 shows results of comparison of MCNP predictions versus actual data for the ${ }^{252} \mathrm{Cf}$ spontaneous fission neutron source. As noted, the overall profile of the data for counts collected versus moderator thickness is in

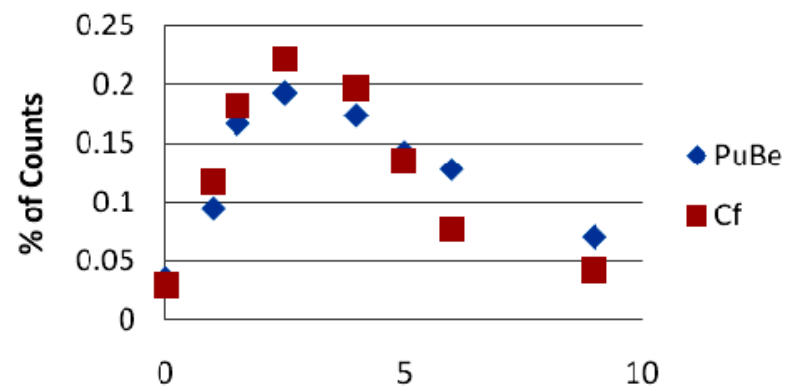

Rectangular moderation thickness (inches)

Figure 9. Experimental data for a LiI detector and ${ }^{252} \mathrm{Cf}$ /Pu-Be source moderated counts vs thickness in "rectangular" geometry.

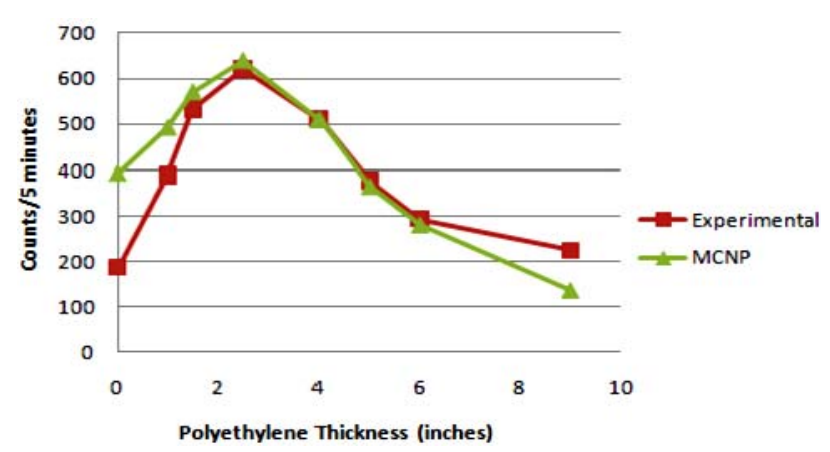

Figure 10. MCNP and experimental data for a LiI detector and ${ }^{252} \mathrm{Cf}$ source moderated in "rectangular" geometry (Figure 3). 
close agreement with predictions. Differences indicate that the model does not (at $\sim 0$ thickness) accurately reflect all of the 3-D intricacies of the experiment geometrical configuration. (e.g., the stand, ceiling, flooring, and intricacies of detector components).

Due to the significant gamma photon emission of ${ }^{252} \mathrm{Cf}$, part of neutron data acquisition is associated with discriminating the counts attributed to gamma photons. Gamma photon interactions result in lower amplitude pulses in thermal neutron detectors compared with pulse heights due to neutron interactions in LiI or $\mathrm{BF}_{3}$ as seen from Figure 11. In Figure 11, the gamma-based and neutronbased counts collected in the multi-channel analyzer (MC A) are shown to occur in channels 1-67, and 115-140, respectively. Counts were generally taken for between 5 and 10 minutes and only the counts from the neutron section of the plots were used to compare with the MCNP predictions. The energy released in the (n, alpha) reaction of a LiI detector is large and such detectors are typically quite good at separation of neutrons and gammas. Liquid Scintillation detectors provide much smaller differences in pulse heights and therefore discriminate via the amount of time that it takes the light pulse to dissipate. Discrimination is at best $95 \%$ effective and much less in a high gamma field. TMFD detectors are gamma insensitive and therefore need not undergo any penalty for separation of counts from neutrons and gammas.

Working with neutron sources requires biological shielding and this shielding can have a significant impact on the count rate of the detector. During the course of the work, it became apparent that there was a greater sensitivity to the environment for "rectangular" than for the "spherical" geometry. In Figure 13, the response of the "spherical", "tent", and "rectangular" geometries have been calculated with MCNP5 for both an ideal geometry as well as a very high moderation geometry made of a table with bricks of paraffin and concrete placed on it (shown in Figure 12). This geometry models the lab table where early experiments were performed before it

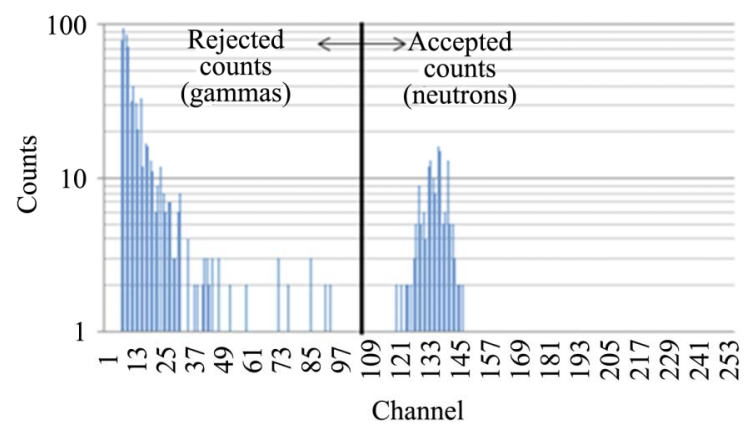

Figure 11. Representative output from LiI detector behind 2.5 inches of polyethylene in "rectangular" geometry exposed to ${ }^{252} \mathrm{Cf}$.

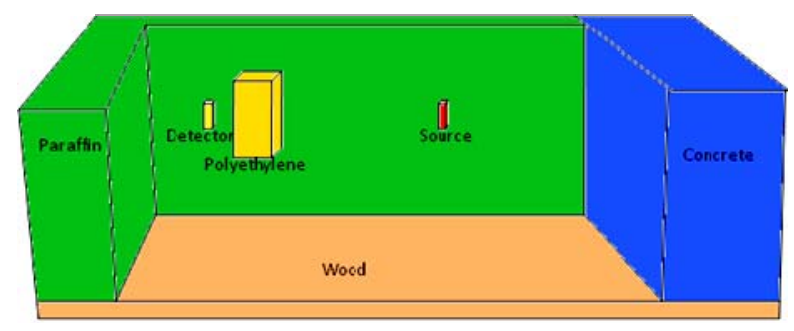

Figure 12. Schematic of the highly moderating environment.

was discovered that the biological shielding had a significant impact on the results of the experiments. For all geometries there is a noticeable difference with and without the presence of the shielding. The difference between curves is much smaller with the "spherical" moderation geometry. Thus, while the "tent" geometry solves some of the problems of low count rate at high energies that the "rectangular" geometry had it does not have all of the same beneficial properties of environmental insulation as the "spherical" moderation geometry.

\section{Selection and Comparison of Unfolding Codes}

While the insights of the response curves from Figures 3, $6,8,9,10$ and 13 are valuable, the ultimate goal of a spectrometry system is to give the neutron source energy spectrum. As mentioned earlier, an unfolding algorithm embodied in a computer code is required for this function. There are several codes that have been developed specifically for Bonner spheres and, in fact, contain common experimentally derived response matrices for common systems as part of the package. The specific codes chosen for this implementation are BON [9], MAXED [12] and GRAVEL [12].

BON is one of the simplest codes available, which uses an unfolding algorithm based on an iterative procedure that converges on the least squares solution [13]. This code is selected for the simplicity of the algorithm as well as the ability to create unbiased if also high variance solutions. An example of a BON unfolding appears in Figure 14. The smooth curve in Figure 14 represents the well-known Watt fission spectrum for neutrons emitted from a spontaneously fissioning ${ }^{252} \mathrm{Cf}$ source. The other curve represents the BON solution spectrum when given data created with MCNP for the response matrix and detector response for a LiI detector in a "rectangular" configuration as discussed earlier in Figure 3. The results are rather accurate for the fast energy bins and somewhat less accurate in the thermal energy bins.

Two other codes assessed were the MAXED and GRAVEL codes [12]. Both of these codes use a priori information about the spectrum in addition to the response matrix and the response to the flux in question. The MAXED code obtains its solution based on maximum entropy 


\section{Low Energy (.065 eV)}

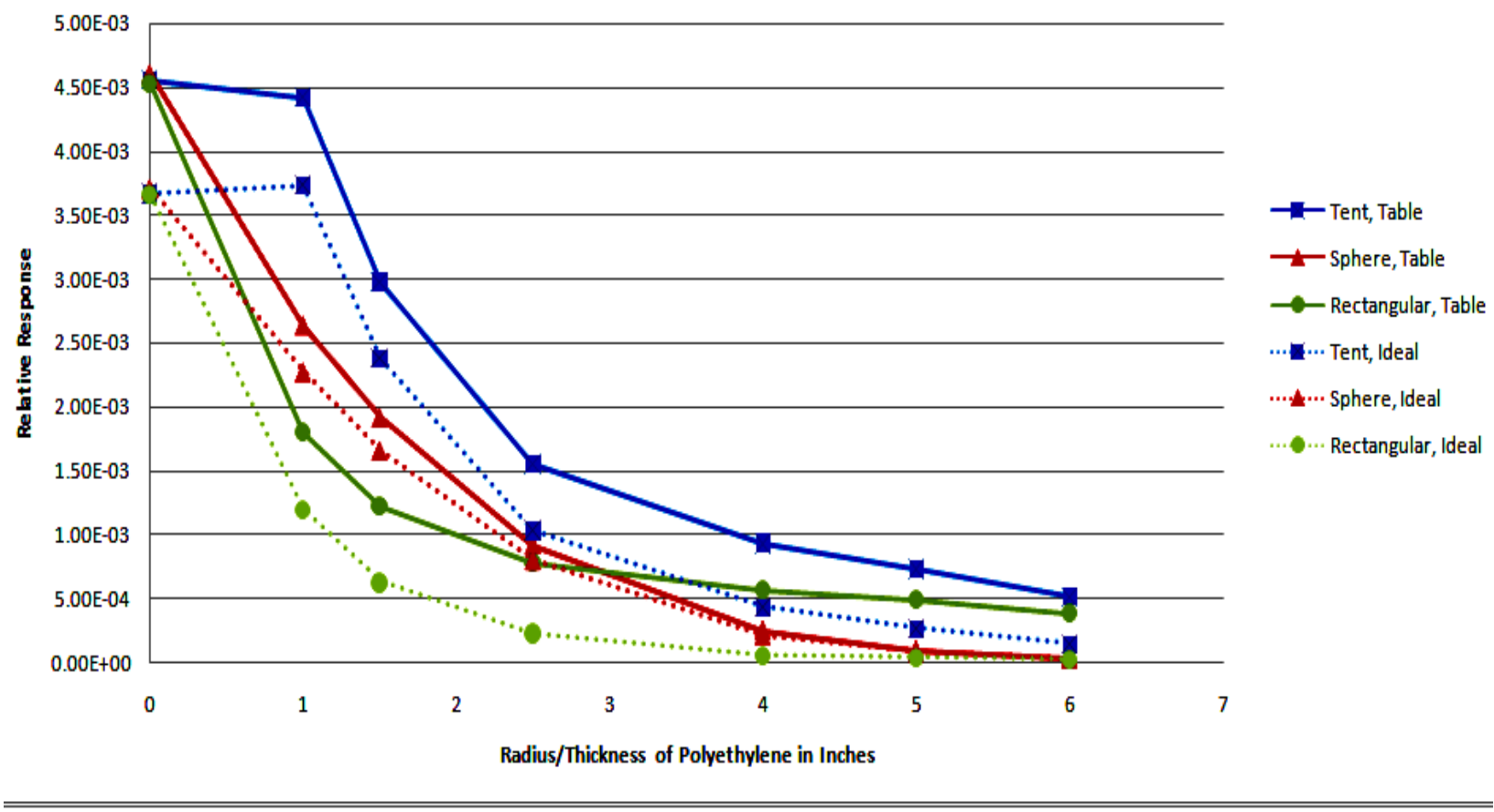

High Energy (5.13 MeV)

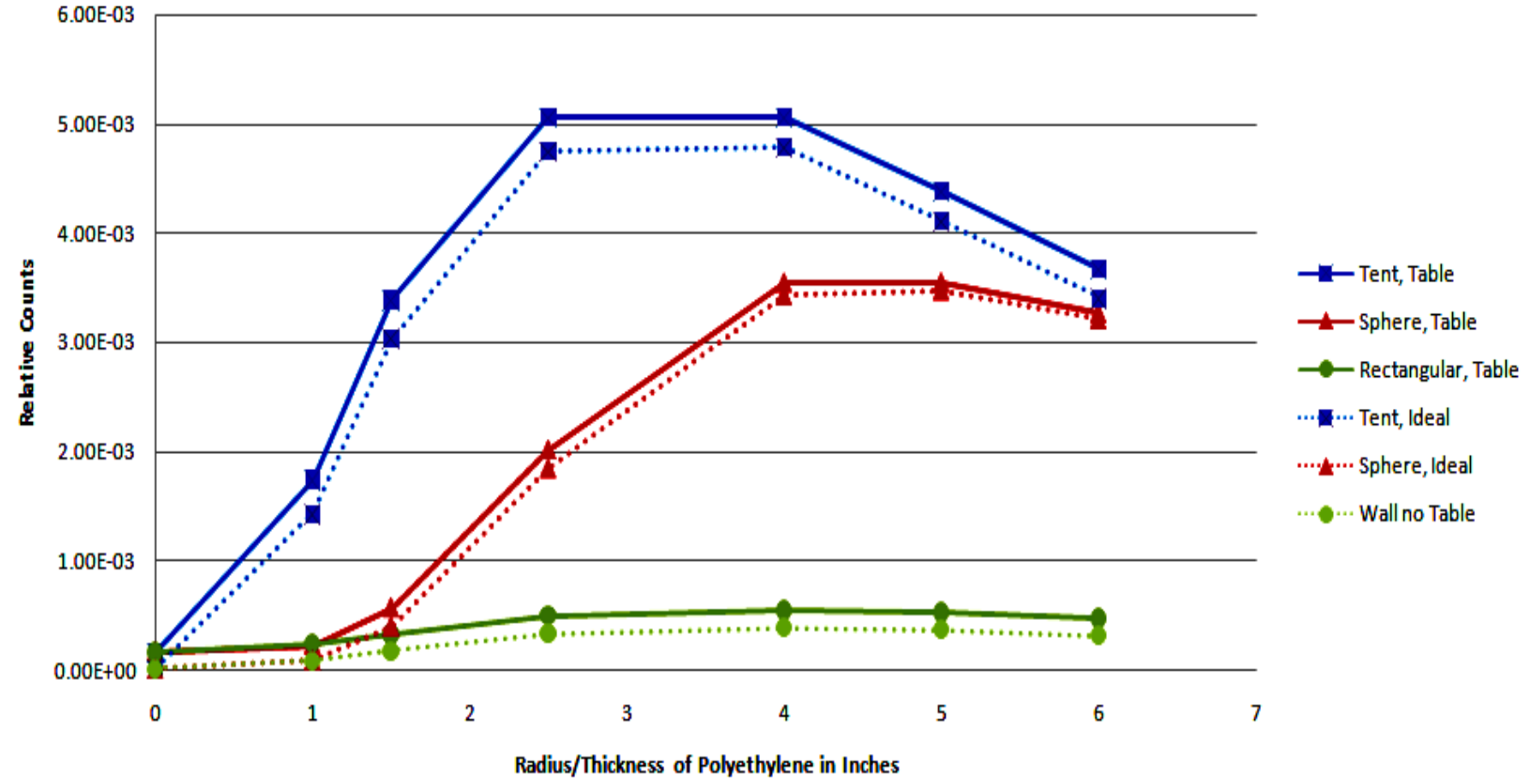

Figure 13. MCNP5 results for proposed geometries in ideal and high moderating environments (Figure 8) with various thicknesses and various incident neutron energies.

methods whereas the GRAVEL code uses iterative methods. These codes were selected because of their ability to deal with some of the spectra that the BON code struggled with as well as the IQU package which came with them that propagates the error and gives the certainty of the output spectrum. Unlike the BON code, the MAXED and GRAVEL codes require a prior information or "guess" spectrum. The guess spectrum is important to the 


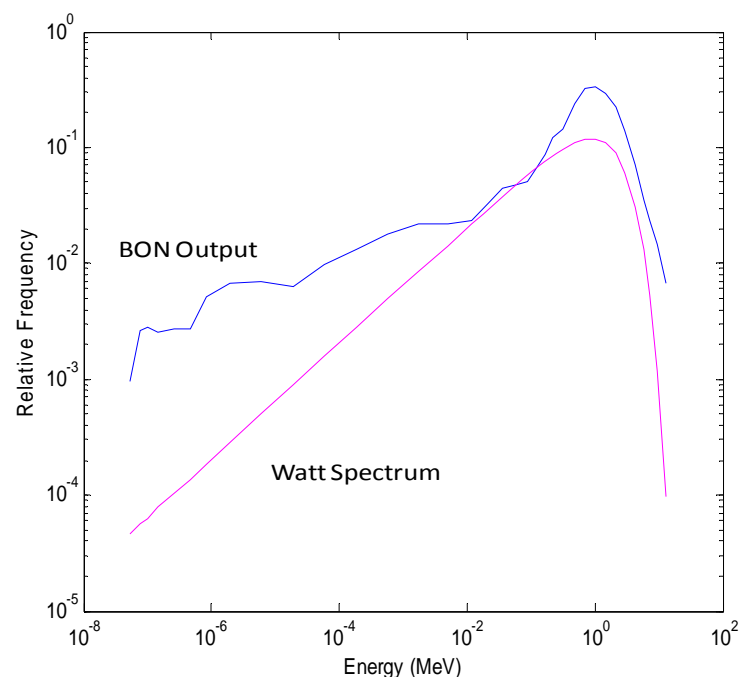

Figure 14. Bon unfolding done on MCNP5 generated data and response matrix for LiI detector in "rectangular" geometry.

final answer that is eventually selected. Figure 15 was produced by using the GRAVEL code when given the input data from the $\mathrm{BF}_{3}$ detector in the "rectangular" geometry. The bad a priori information curve is the program output when a flat line is put in as the guess spectrum and the good a priroi information curve is the program output when the Watt Spectrum is put in as the guess spectrum. Nevertheless, even with bad a priori information, the result is very close for the relatively more important bins in the higher energy range (i.e., above $0.01 \mathrm{MeV}$ ).

\section{Application to TMFD}

TMFD spectroscopy has the potential to revolutionize the field of spectroscopic detectors: the TMFD's extremely high intrinsic efficiency makes the detectors ideal for low fluence scenarios, commonplace in passive interrogation; the complete gamma blindness [3,6] makes these detectors ideal for the high-background environment of a reprocessing stream, and the drive amplitude modification of the detector response function makes data acquisition much simpler.

\subsection{Moderation Based Spectroscopy}

Figure 16 [14] shows several curves for the waiting time response of the CTMFD (with trimethyl-borate as the metastable detector liquid) when it is exposed to different neutron fluxes from an isotope source. The first curve is for the source 4 "away, the second is for a source 20" away, the third is for a source 20 "away with 8 " of paraffin

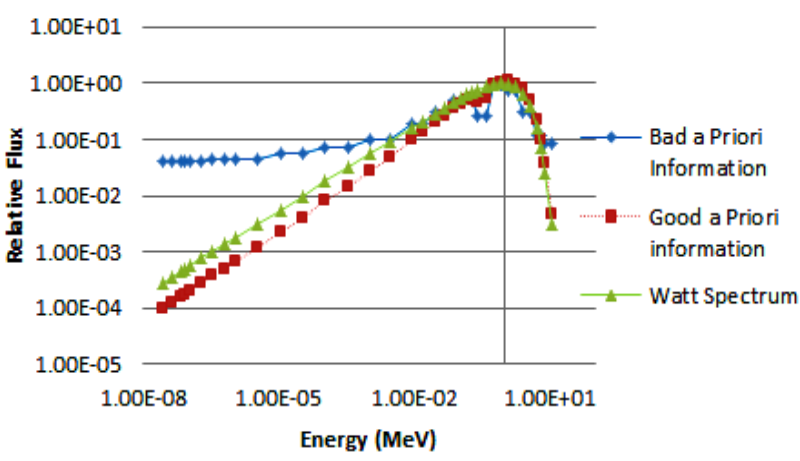

Figure 15. GRAVEL unfolding for MCNP5 generated counts and response matrix for $\mathrm{BF}_{3}$ detector in "rectangular" geometry with ${ }^{252} \mathrm{Cf}$ source. Shown are GRAVEL unfoldings with two different qualities of a priori information and the true Watt fission spectrum.

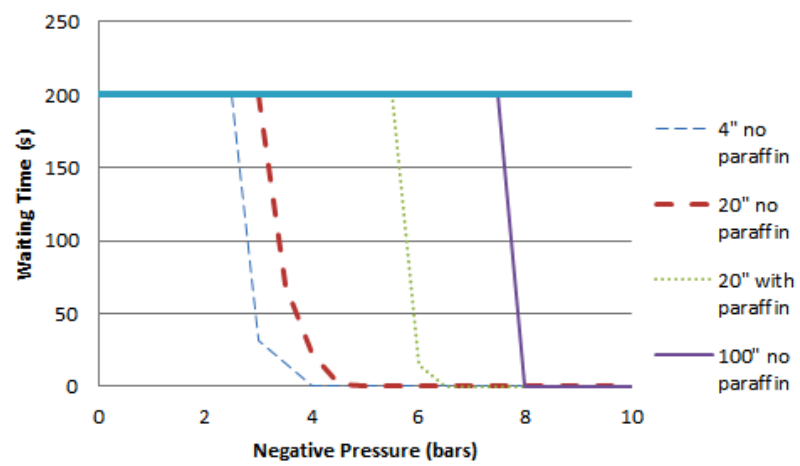

Figure 16. Waiting time curves for Trimethyl-Borate filled detector [14].

shielding between the source and the detector, and the fourth is for a source 100" away. For the curves without paraffin shielding, increasing the negative pressure increases the percentage of neutrons that produce effective collisions and lowers the wait time.

For the curve with the paraffin shield, it can be assumed that virtually all of the neutrons are down-scattered to the $\mathrm{eV}$ range, after scattering through 8 " of paraffin. Thus, there is a distinct range of negative pressures where the alpha particles from the ${ }^{10} \mathrm{~B}(\mathrm{n})$ reactions become effective at creating detection events and there is a very sharp decrease in waiting time as all of these reactions start causing the onset of rapidly (within nanoseconds) forming and growing cavitation nuclei in the fluid.

These curves demonstrate that the principle of correlating waiting time to negative pressure and neutron flux intensity has been proven. Thus, there is a practical possibility to use a thermal neutron approach of down-scattering neutrons to the sub-eV range, detecting via (n) 
reaction and unfolding the result with BON, MAXED and/or GRAVEL.

\subsection{CTMFDs Speed Based Spectroscopy}

Rather than introducing a moderating material in order to change the neutron sensitivity of the TMFD system, it is excitingly possible to change on demand, the amount of negative pressure being applied and thus change the sensitivity of the system to various energies of neutrons. Because bubble nucleation requires a minimum amount of energy in order to proceed, only neutrons above a threshold will be able to cause detection events and those near/below the threshold will do so with very low probability.

Because of this effect, neutron fluences with different energy spectra cause detection events at different rates when the detector is brought to different negative pressures. Figure 17 shows the different responses of a R113 filled CTMFD to various neutron spectra.

Different working fluids will respond to neutrons at very different negative pressures. Cavitation thresholds with fluids impinged by PuBe neutrons are anywhere between 1 and 12 bar for most organic fluids.

Data obtained from the same detector at different levels of metastability could be unfolded to find the neutron spectrum in much the same way that data from different moderation geometries can be used.

\subsection{Drive Amplitude Based Spectroscopy}

Especially in the ATMFD, and to some lesser degree in the CTMFD, the amount of energy used for achieving the desired tension metastable state will change the volume that becomes sensitive to neutrons. Because the detectors are filled with fluid that is designed to scatter neutrons and remove large portions of the neutron energy, there will be a change in the spectrum that reaches the sensitive portion of the detector when there is a change in drive power. This change increases the amount of spectroscopic information that is available while changing metastable states of the detector.

\subsection{Theoretical Response Matrix Calculation}

Because providing monoenergetic neutron sources is both difficult and prohibitively expensive, it is desirable to be able to determine the response matrix of a TMFD using Monte Carlo methods much like what was done for the thermal neutron detectors using Bonner Spheres. It is possible to characterize the geometry and determine the distribution of energy deposited by neutron scatters. It is equally possible to know the distribution of negative

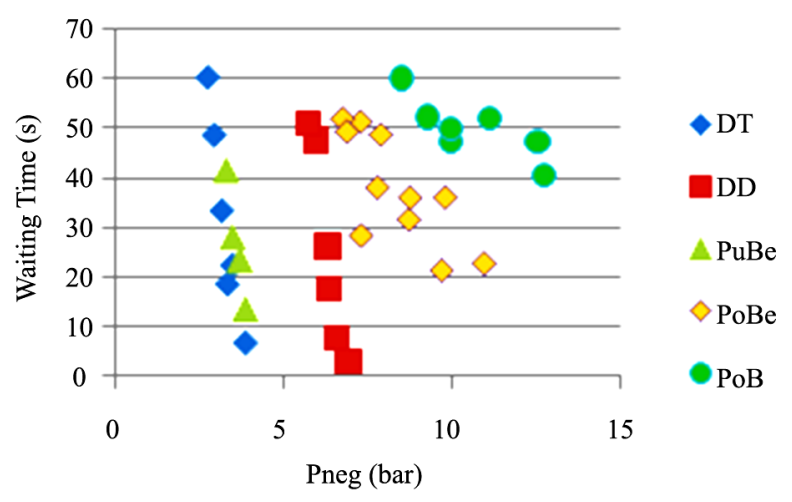

Figure 17. Waiting time curves for a CTMFD with R113 as the working fluid.

pressure (especially in the CTMFD). Unfortunately, the physical mechanisms for detection via nucleation in a sub-zero pressure state within TMFD systems is vastly more complex than it is in the thermal neutron detectors. Prevailing theory for bubble nucleation criteria in tensioned metastable states misses the mark by orders of magnitude [15] making it unsuitable for modeling detector response. Further fundamental studies are underway to better characterize bubble nucleation parameters, and thus, to make it possible to determine the response matrix of a TMFD system through first-principles theoretical modeling.

\section{Summary and Conclusions}

This section presents a summary of the work performed accompanied with concluding remarks in various subsections.

\subsection{Conventional (LiI,_BF3,_3He)_Detector Based Spectroscopy}

The MCNP-model based approach (described in this paper) together with unfolding codes such as BON, MAXED and GRAVEL has been shown capable to provide incoming neutron energy spectral information not just for the conventional "spherical" moderator geometries, but now also for generalized "non-spherical" geometrical configurations. Using our described procedure, neutron spectral information can be derived with use of relatively inexpensive (as much as 100 times less) moderator sheets of varying thickness tailored to the spectrum and the detector rather than specially machined Bonner spheres. The validation exercises have proven successful when compared with past computations of others in "spherical" geometry, and thereafter, also against direct experimental data we obtained with ${ }^{252} \mathrm{Cf}$ and $\mathrm{Pu}-\mathrm{Be}$ isotope neutron sourcess in "non-spherical" moderator configurations. It was clear that, for "non-spherical" geometries, it is even 
more important to model the full 3-D aspects of the system than with conventional "spherical" moderation. Finally, a new moderation geometry has been introduced in the form of the "tent" configuration in order to serve as a guideline for deriving directly usable results from nonstandard moderation geometries.

\subsection{CTMFD Based Spectroscopy}

It has already been demonstrated that a CTMFD filled with borated liquid is sensitive to thermal neutrons in much the same way as a conventional thermal neutron detector would be and thus can carry out spectrometry in much the same way. More excitingly, the negative pressure vs. particle energy characteristics of the system have been confirmed as well and thus the detector's sensitivity to neutrons could be tailored by different operational modes rather than by moderation-ultimately making for a much better system, particularly for low flux scenarios. As such, CTMFD systems may be ideal for port area monitoring and spectroscopy.

\subsection{ATMFD Based Spectroscopy}

The ATMFD system permits the user to tailor the volume of sensitivity to neutrons at will. As a consequence, the same ATMFD volume can be induced to provide differing levels of "self" moderation of incoming neutrons. Such an approach completely dispenses with the need to position moderator blocks external to the detector. There would be significant improvement in detection time over CTMFD systems, but as a tradeoff, the system would suffer from a larger (but not insurmountable) amount of complexity in deriving the response matrix. This system may be the most desirable for area monitoring of high flux environments such as material accountability of waste spent nuclear fuel processing streams.

\section{Acknowledgements}

Support for this research was provided from the United States National Science Foundation for which co-author Tom Grimes is a fellowship recipient and $\mathrm{PhD}$ student in Professor Taleyarkhan's research group at Purdue University. Additional support was provided by the United States Department of Energy, the Department of Homeland Security, and Purdue University. Assistance from laboratory colleagues and Purdue's Radiological and Environmental Management Services is acknowledged.

\section{References}

[1] DHS DNDO Domestic Nuclear Detection Office Mission. http://www.dhs.gov/xabout/structure/editorial_0766.shtm

[2] B. B. Cipiti, "Advanced Instrumentation for Reprocessing," Technical Report SAND2005-6223, Sandia National Laboratories, Albuquerque, October 2005.

[3] R. Bean, "Aqueous Processing Material Accountability Instrumentation,” Technical Report INL/EXT-07-13431, Idaho National Laboratory, Idaho, September 2007.

[4] A. Sansone, S. Zielinski, J. A. Webster, J. Lapinskas, R. P. Taleyarkhan and R. C. Block, "Gamma-Blind Nuclear Particle-Induced Bubble Formation in Tensioned Metastable Fluids," ANS 2011 Conference, Holywood, 2011.

[5] R. P. Taleyarkhan, J. R. Lapinskas and Y. Xu, "Tension Metastable Fluids and Nanoscale Interactions with External Stimuli-Theoretical-Cum-Experimental Assessments and Nuclear Engineering Applications," Nuclear Engineering and Design, Vol. 238, No. 7, 2008, pp. 1820 -1827. doi:10.1016/j.nucengdes.2007.10.019

[6] B. Archambault, J. A. Webster, J. R. Lapinskas, T. F. Grimes, R. P. Taleyarkhan and A. Eghlima, "Transformational Nuclear Sensors-Real-Time Monitoring of WMDs, Risk Assessment \& Response,” IEEE 2010.

[7] Taleyarkhan, et al., "Tensioned Metastable Fluids and Nanoscale Interactions," NED, Vol. 238, No. 7, July 2008, pp. 1820-1827.

[8] G. Knoll, "Radiation Detection and Measurement," 3rd Edition, John Wiley \& Sons, New York, 2000.

[9] R. S. Sanna, "A Manual for BON: A Code for Unfolding Multisphere Spectrometer Neutron Measurements," EML -394, August 1981.

[10] J. F. Briesmeister, "MCNP ${ }^{\mathrm{TM}}$-A general Monte Carlo NParticle Transport Code," Los Alamos National Laboratory Report LA-13709-M, Los Alamos, 2000.

[11] H. R. Vega-Carrillo, et al., "BSS/6LiI Response Matrix," LAS/ANS Symposium, Cancun, 1-5 July 2007.

[12] M. Reginatto, “The 'Few-Channel' Unfolding Programs in the UMG Package: MXD_FC33, GRV FC33 and IQU_FC333," Physikalisch-Technische Bundesanstalt (P TB), Braunschweig, 1 March 2004.

[13] G. Rabmond, "An Iterative Unfolding Method for Response Matricies," ANL-6984, Argonne National Lab, Argonne, December 1964.

[14] Lapinskas, et al., "Tension Metastable Fluid Nuclear Particle Detector-Qualification and Comparisons," Proceedings of ICONE 16-Paper \#48185, Orlando, 11-15 May 2008.

[15] R. P. Taleyarkhan, C. D. West and J. S. Cho, "Energetics of Nano-to-Macro Scale Triggered Tensioned Metastable Fluids ORNL/TM-2022/233," Oak Ridge National Lab, Oak Ridge, November, 2002.

[16] J. Lapinskas, B. Archambault, J. Webster and S. Zilenski, "Towards Leap-Ahead Advances in Radiation Detection," ICONE 2008, Oral Presentation, Orlando, 11-15 May 2008 